\title{
Foundation of quantum mechanics from the principle of relativity
}

\author{
Laurent Nottale \\ CNRS, LUTH, Observatoire de Paris-Meudon, F-92195 Meudon Cedex, France
}

\begin{abstract}
We briefly recall the main steps by which we suggest to found quantum mechanics and gauge field theories on the principle of relativity, once it is extended to scale transformations of the reference system. The wave functions are constructed as consequences of the nondifferentiability of a continuous space-time, while the Schrödinger and Dirac equations are obtained from its geodesics equations. In this framework, the gauge fields emerge as manifestation of the fractal geometry, and the gauge charges as the conservative quantities which are built from its internal symmetries.
\end{abstract}

Keywords: Scale relativity, Foundation of quantum mechanics, Gauge field theories PACS: 03.65.Ta, w1.15.-q

\section{INTRODUCTION}

In the theory of scale relativity, one gives up the hypothesis of space-time differentiability. One can prove that a nondifferentiable continuum is fractal [1,2], i.e., it is explicitly dependent on the scales of resolution. This leads one to extend the principle of relativity to scale transformations. In the present contribution, we summarize the steps by which one recovers, in this framework, the main postulates of quantum mechanics and of gauge field theories. A more detailed account can be found in Refs. [6, 10, 14, 15].

\section{QUANTUM MECHANICS IN SCALE RELATIVITY}

From the three main consequences of nondifferentiability [7], namely, (i) infinite number of geodesics, (ii) breaking of the reflexion invariance of the time differential element $d t$ that leads to a two-valuedness of the velocity field, (iii) fractal dimension 2 of the geodesics, one describes the elementary displacements $d X$ on a fractal space $[1,3,4,5]$ as $d X_{ \pm}=d_{ \pm} x+d \xi_{ \pm}$, where $d \xi$ is the "fractal part" and $d x$ the "classical part",

$$
d_{ \pm} x=v_{ \pm} d t, d \xi_{ \pm}=\eta \sqrt{2 \mathscr{D}} d t^{1 / 2}
$$

Here $\eta$ is a stochastic dimensionless variable such that $\langle\eta\rangle=0$ and $\left\langle\eta^{2}\right\rangle=1$, and $\mathscr{D}$ a parameter that generalizes the Compton scale. The two time derivatives are then combined in a complex derivative [1], $\widehat{d} / d t=\left(d_{+}+d_{-}\right) / 2 d t-i\left(d_{+}-d_{-}\right) / 2 d t$. Applying this operator to the position vector yields a complex velocity $\mathscr{V}=\widehat{d x} / d t$. Then

\footnotetext{
1 To appear in: Proceedings of the Eight International Symposium Frontiers of Fundamental Physics, Madrid, Spain, 17-19 October 2006, Eds. Sidharth B.G. et al., A.I.P. Conf. Proc., in press.
} 
the geodesics equation, $\widehat{d} \mathscr{V} / d t=0$, can be integrated under the form of a generalized Schrödinger equation $[1,2,6,9]$. We have recently generalized the proof to the whole velocity field $\widetilde{\mathscr{V}}=\mathscr{V}+\mathscr{W}$, including its differentiable and nondifferentiable parts [13, 10]. We can build from it a full complex action, $d \tilde{\mathscr{S}}=(1 / 2) m(\mathscr{V}+\mathscr{W})^{2} d t$, then define a full wavefunction as $\tilde{\psi}=\exp (i \tilde{\mathscr{S}} / 2 m \mathscr{D})$, such that $\tilde{\mathscr{V}}=\mathscr{V}+\mathscr{W}=\nabla \tilde{\mathscr{S}} / m=$ $-2 i \mathscr{D} \nabla \ln \tilde{\psi}$. In our framework, this relation keeps a mathematical and physical meaning in terms of fractal functions, explicitly dependent on $d t$ and divergent when $d t \rightarrow 0$. Then one builds a generalized full covariant derivative, that reads [10]

$$
\frac{\widehat{d}}{d t}=\frac{\partial}{\partial t}+(\mathscr{V}+\mathscr{W}) \cdot \nabla-i \mathscr{D}(1+\tilde{\zeta}) \Delta
$$

where $\tilde{\zeta}$ is a stochastic variable of zero mean. Using this covariant derivative, we can finally write a covariant equation which keeps the form of Newton's fundamental equation of dynamics,

$$
\frac{\widehat{d}}{d t} \widetilde{\mathscr{V}}=-\frac{\nabla \phi}{m}
$$

This equation can be integrated in terms of a generalized Schrödinger equation $[13,10]$,

$$
\mathscr{D}^{2} \Delta \tilde{\psi}+i \mathscr{D} \frac{\partial \tilde{\psi}}{\partial t}-\frac{\phi}{2 m} \tilde{\psi}=0
$$

which now allows fractal solutions, in agreement with Berry's [11] and Hall's [12] results. But this property is obtained here as a manifestation of space nondifferentiability.

The von Neumann's and Born's postulates are then derived from the identification of "particles" with the various geometric properties of fractal space-time geodesics $[6,10]$.

Then the account of a new two-valuedness, consequence of nondifferentiability, leads to introduce bispinors in terms of biquaternionic wavefunctions, and to obtain the Dirac [6] and Pauli [14] equations as geodesics equations of a fractal space-time.

\section{GAUGE THEORIES IN SCALE RELATIVITY}

The theory of scale relativity can also be applied to the foundation of Abelian $[2,8]$ and non-Abelian [15] gauge theories. This application is based on a general relativistic description of the internal structures of nondifferentiable space-time geodesics in terms of tensorial scale variables $\eta_{\alpha \beta}(x, y, z, t)$ which may now be function of the coordinates.

We assume for simplicity that the two tensorial indices can be gathered under one common index. The infinitesimal transformation law on the $\eta_{\alpha}$ can be written in a linear way as $\eta_{\alpha}^{\prime}=\left(\delta_{\alpha \beta}+\delta \theta_{\alpha \beta}\right) \eta^{\beta}$. Since the $\eta_{\alpha}$ 's are now functions of the standard spacetime coordinates, this leads us to define a new scale-covariant derivative,

$$
d \eta_{\alpha}=D \eta_{\alpha}-\eta^{\beta} \delta \theta_{\alpha \beta}=D \eta_{\alpha}-\eta^{\beta} W_{\alpha \beta}^{\mu} d x_{\mu}
$$

The gauge potentials $W_{\alpha \beta}^{\mu}$ naturally emerge from this geometrical framework. 
The next step amounts to describe how various physical quantities transform under these $\eta_{\alpha}$ transformations. To this purpose we generalize to multiplets the relation $\mathscr{V}_{\mu}=$ $i \lambda \psi^{-1} \partial_{\mu} \psi$ obtained in the Dirac spinor case [6]:

$$
\mathscr{V}_{j k}^{\mu}=i \lambda \psi_{j}^{-1} \partial^{\mu} \psi_{k}
$$

The action $d S_{j k}=d S_{j k}\left(x^{\mu}, \mathscr{V}_{j k}^{\mu}, \eta_{\alpha}\right)$ also becomes a biquaternionic tensor, so that

$$
\partial^{\mu} S_{j k}=D^{\mu} S_{j k}-\eta^{\beta} \frac{\partial S_{j k}}{\partial \eta_{\alpha}} W_{\alpha \beta}^{\mu}
$$

This result allows one to define a general non-Abelian group of scale transformations whose generators are $T^{\alpha \beta}=\eta^{\beta} \partial^{\alpha}$, yielding the generalized charges,

$$
\frac{\tilde{g}}{c} t_{j k}^{\alpha \beta}=\eta^{\beta} \frac{\partial S_{j k}}{\partial \eta_{\alpha}}
$$

Knowing that the $\alpha, \beta$ represent two indices each, this is a large group that contains the standard model $U(1) \times S U(2) \times S U(3)$ as subset [15].

As we have shown in more detail in Ref. [15], the various ingredients of Yang-Mills theories can be recovered in such a framework, but they are now founded on geometric and scale-relativistic first principles.

\section{CONCLUSION}

In this contribution, we have recalled the main steps that lead to a new foundation of quantum mechanics and of gauge fields on the principle of relativity itself, once it is generalized to scale transformations of the reference system.

For this purpose, two covariant derivatives have been constructed, which account for the nondifferentiable and fractal geometry of space-time, and which allow to write the equations of motion as geodesics equations. After change of variable, these equations finally take the form of the quantum mechanics and quantum field equations.

\section{ACKNOWLEDGMENTS}

I gratefully acknowledge Dr. Sidharth for his kind invitation to the FFP8 symposium.

\section{REFERENCES}

1. L. Nottale, Fractal Space-Time and Microphysics, World Scientific, Singapore, 1993.

2. L. Nottale, Chaos, Solitons \& Fractals 7 (1996), 877.

3. G. N. Ord, J. Phys. A: Math. Gen. 16 (1983), 1869.

4. L. Nottale and J. Schneider, J. Math. Phys. 25 (1984), 1296.

5. L. Nottale, Int. J. Mod. Phys. A 4 (1989), 5047.

6. M.N. Célérier \& L. Nottale, J. Phys. A: Math. Gen. 37 (2004), 931. 
7. R. P. Feynman and A. R. Hibbs, Quantum Mechanics and Path Integrals, MacGraw-Hill, 1965.

8. L. Nottale, in Relativity in General, Diaz Alonso \& Lorente Paramo eds., Frontières, 1994, p. 121.

9. J. Cresson, J. Math. Phys. 44 (2003), 4907.

10. L. Nottale, in Proc. 7th Int. Coll. on Clifford Algebra, Ed. P. Anglès, Birkhauser, (2006) in press

11. M.V. Berry J. Phys. A: Math. Gen. 29 (1996), 6617.

12. M.J.W. Hall J. Phys. A: Math. Gen. 37 (2004) 9549.

13. L. Nottale, Chaos, Solitons \& Fractals 10 (1999), 459.

14. M.N. Célérier \& L. Nottale, J. Phys. A: Math. Gen. 39 (2006),12565.

15. L. Nottale, M.N. Célérier \& T. Lehner, J. Math. Phys. 47 (2006), 032303. 